\title{
Abordaje metodológico en salud sexual y reproductiva en situaciones de discapacidad con equipos de salud en Uruguay
}

\section{Methodological approach in sexual and reproductive health in situations of disability with health teams in Uruguay}

\section{Palabras clave}

Discapacidad, salud sexual y reproductiva, servicio de salud, ciudadanía.

\section{Keywords}

Disability, sexual and reproductive health, health care, citizenship.

\author{
María Carolina Farías \\ Rodríguez \\ <cfarias@psico.edu.uy>
}

Universidad de la República. Uruguay

\author{
Julia Córdoba Wolman \\ <jcordoba@psico.edu.uy> \\ Universidad de la República. Uruguay
}

Eugenia Barbosa Molina

<meugenia.bar@psico.edu.uy>

Universidad de la República. Uruguay

\section{Gonzalo Gelpi Pepe}

<ggelpi@psico.edu.uy>

Universidad de la República. Uruguay

\section{Cecilia Caillet-Bois Duvoy \\ <ccailletbois@gmail.com>}

Universidad de la República. Uruguay

\section{Alejandra López Gómez}

<alopez@psico.edu.uy>

Universidad de la República. Uruguay

María José Bagnato Núñez <jcordoba@psico.edu.uy>

Universidad de la República. Uruguay

Según el Informe Mundial sobre la Discapacidad más de mil millones de personas viven en todo el mundo con alguna forma de discapacidad (o sea, alrededor del $15 \%$ de la población mundial según las estimaciones de la población mundial en 2010); de ellas, casi 200 millones experimentan dificultades considerables en su funcionamiento. De hecho, en los años futuros, la discapacidad será un motivo de preocupación aún mayor porque su prevalencia va en aumento (Organización Mundial de la Salud, 2011). En Uruguay esta temática tiene especial relevancia y alcance: en el año 2011,

\footnotetext{
1. Por Naciones Unidas en Uruguay participaron: ONU Mujeres, OPS/OMS (Organización Panamericana de la Salud) y UNFPA (Fondo de Población de las Naciones Unidas). Por el Estado Uruguayo: Administración de los Servicios de Salud del Estado (ASSE), Agencia Uruguaya de Cooperación Internacional (AUCI), Banco de Previsión Social (BPS), Ministerio de Desarrollo Social (MIDES) con sus Secretarías del Programa Nacional de Discapacidad (PRONADIS) y el Instituto Nacional de las Mujeres (INMUJERES), Instituto Nacional de Estadística (INE) y el Ministerio de Salud Pública (MSP). Por la Sociedad Civil participó la Alianza de Organizaciones por los Derechos de las Personas con Discapacidad en Uruguay.
}

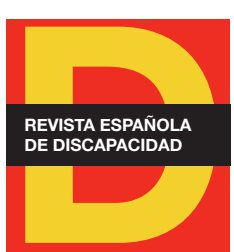

Para citar:

Farías, M. C. (2021). Abordaje metodológico en salud sexual y reproductiva en situaciones de discapacidad con equipos de salud en Uruguay. Revista Española de Discapacidad, 9(1), pp. 261-271.

Doi: <https://doi.org/10.5569/23405104.09.01.14> 
las personas que tenían al menos una limitación, eran en torno al $16 \%$ de la población total (Instituto Nacional de Estadística de Uruguay, 2011).

En el año 2010, Uruguay aprobó la Ley 18.651 Protección integral de personas con discapacidad, reconociendo un reclamo de larga data de las organizaciones de la sociedad civil. Esta ley debe ubicarse en un marco de importantes y estratégicas transformaciones del Estado uruguayo que tuvo lugar entre 2005 y 2020. En 2008 se creó el Sistema Nacional Integrado de Salud a través de la Ley 18.211 (2008). La reforma de salud que consagró esta ley incluyó cambios en el modelo de atención, de financiación y de gestión, siguiendo los principios rectores de universalidad, accesibilidad, equidad, calidad de la atención, promoción y prevención integral (Ministerio de Salud Pública, 2010). El Sistema Nacional Integrado de Salud prioriza el primer nivel de atención en el que se resuelve la amplia mayoría de los problemas de salud (Vignolo et al., 2011). Dicho nivel es el contacto inicial que los usuarios tienen con el sistema sanitario, por lo que tiene la potencialidad de ser una puerta de entrada al sistema o la primera barrera que las personas usuarias deben enfrentar, si se trata de servicios que reproducen situaciones de discriminación.

En el mismo año se aprobó la Ley 18.335 Pacientes y usuarios de los servicios de salud, que estableció que los usuarios y usuarias de salud tienen derecho a un trato igualitario y sin discriminación, y a una atención de calidad de la mano de profesionales debidamente capacitados. También se aprobó la Ley 18.426 de Defensa de la salud sexual y reproductiva, la cual establece que los derechos sexuales y los derechos reproductivos son derechos humanos reconocidos por el Estado uruguayo. Este marco normativo motivó la realización de una guía técnica en salud sexual y reproductiva para los servicios de salud, que incluye consideraciones específicas para las personas en situación de discapacidad (Ministerio de Salud Pública, 2010). El documento contó con el apoyo del Ministerio de Salud Pública (MSP), el Programa Nacional de Discapacidad (PRONADIS) dependiente del Ministerio de Desarrollo Social, la Intendencia de Montevideo (IM), ONU Mujeres, el Fondo de Población de las Naciones Unidas (UNFPA) y la Organización Panamericana de la Salud (OPS). El documento ofrece un marco teórico de referencia y recomendaciones tanto para los equipos técnicos como para el sistema de salud (Mattioli, 2012). Finalmente, en el año 2012, se aprueba la Ley 18.987 de interrupción voluntaria del embarazo, transformándose así en uno de los pocos países de América Latina en contar con leyes que reconocen este derecho. La agenda de derechos y género en Uruguay se completa con otras leyes que, consideradas en su conjunto, constituyen un marco jurídico destacado en la región y a nivel internacional.

El Sistema Nacional Integrado de Salud está conformado por 45 instituciones prestadoras de servicios integrales públicos y privados, distribuidos en todo el territorio nacional. En cuanto a la cobertura, el 59,4\% de la población cuenta con seguro privado y mutual, mientras que, el 40,6\% con seguro público (Registro Único de Cobertura de Asistencia Formal, 2018). Con excepción de la Sanidad Militar, en el resto de prestadores de salud, las mujeres lideran las afiliaciones (Registro Único de Cobertura de Asistencia Formal, 2018).

En 2015, el Estado uruguayo fijó objetivos sanitarios estratégicos: 1. alcanzar mejoras en la situación de salud de la población; 2. disminuir las desigualdades en el derecho a la salud; 3. mejorar la calidad de los procesos asistenciales de salud; 4. generar las condiciones para que las personas tengan una experiencia positiva en la atención de su salud. Estos objetivos se relacionan directamente con las necesidades y demandas del colectivo de personas en situación de discapacidad. Además, se definieron los Objetivos Sanitarios Nacionales 2020 relacionados con dificultades de acceso a servicios en personas con discapacidad y atención a adultos mayores vulnerables, violencia de género y generaciones, embarazo no deseado en adolescentes, alteraciones del desarrollo en la primera infancia, lesiones y muertes por siniestralidad vial y en el ámbito laboral (Ministerio de Salud Pública, 2019). Algunos de los objetivos predominantemente 
podrían referir a situaciones de discapacidad congénita, otros a situaciones de discapacidad adquirida y el resto a consecuencias específicas de vivir en situación de discapacidad.

Palacios (2016) plantea que para garantizar que todas las personas puedan hacer uso de los servicios y espacios en una comunidad es necesario pensar entornos con diseños universales y garantizar a las personas ajustes que compensen limitaciones funcionales. Abordar la salud de las personas con discapacidad desde esta mirada compleja depende de la formación del personal de la salud, así como de los respaldos técnicos y legales disponibles (Rudolf et al., 2009).

Los marcos normativos internacionales y nacionales promueven el diseño de políticas públicas inclusivas, que incorporen el acceso a una atención integral de la salud de las personas en situación de discapacidad. En esta línea, los aportes de las organizaciones de la sociedad civil y del ámbito académico, han tenido un rol fundamental en colocar en el debate público temas vinculados a la humanización de la atención y la asistencia, la promoción y prevención de la salud y la protección de los derechos relativos al acceso a la salud (incluyendo la salud sexual y reproductiva).

La sexualidad es una dimensión central en las personas a lo largo del curso de vida y se encuentra en la base de los procesos de salud-enfermedad. En tanto producción socio histórica, la sexualidad, así como la salud sexual y reproductiva son campos de disputa político, social, ideológica, jurídica, ética (López, 2013). Su reconocimiento como dimensión de derechos humanos es parte de la conquista de los movimientos de mujeres y de la diversidad sexual. Un desarrollo sexual saludable depende de la satisfacción de las necesidades básicas humanas como son el deseo de contacto, la intimidad, la expresión emocional, el placer, la ternura y el amor. Para poder mantener la salud sexual es necesario que se reconozcan y defiendan los derechos sexuales de todas las personas (Organización Panamericana de la Salud y Organización Mundial de la Salud, 2013). Históricamente ha existido un tabú asociado a la sexualidad de las personas en situación de discapacidad y ha predominado el silencio, porque poner en palabras las experiencias sexuales de este grupo suele generar incomodidad y rechazo social (Nagai y Pereira, 2017). Se han asignado a las personas con discapacidad preconceptos negativos, como por ejemplo: ser asexuados, perversos, "infantiles", que tienen disfunciones sexuales, que son incapaces de mantener un vínculo afectivo-sexual, que no tienen capacidad para el ejercicio de la maternidad y paternidad, que no precisan información relativa a la sexualidad, entre otros (Mattiolli, 2012). Estos preconceptos obstruyen la capacidad de reconocerles como sujetos sexuados y sujetos de derechos.

La forma de pensar, sentir y actuar de mujeres y varones tiene matices que se asocian a los permisos y las prohibiciones que se otorgan a partir de las normas de género en una cultura y en un momento sociohistórico dado. A medida que las personas se desarrollan, generan la capacidad de revisar sus creencias de género y construir una identidad genérica más cercana o más lejana de los modelos hegemónicos disponibles de masculinidad y feminidad (López et al., 2015). El género, así como la discapacidad, no son sólo categorías de análisis social sino que se interseccionan con otras desigualdades sociales como la clase social, la etnia/raza, la nacionalidad, y con otras dimensiones como la orientación sexual y/o la identidad de género y la edad. En el caso de las personas en situación de discapacidad, las cuestiones de género suelen perjudicar más especialmente a las mujeres de distintas condiciones y procedencias.

A pesar de los avances en la inclusión de la discapacidad en la agenda de las políticas públicas y en el debate público, el reconocimiento social de los derechos de las personas en situación de discapacidad es un proceso que requiere de acciones sostenidas. En tal sentido, la intervención que se desarrolló buscó contribuir a la incorporación de buenas prácticas por parte del personal (técnico y administrativo) en relación a la atención de la salud de las personas en situación de discapacidad y sus familias. 


\section{Objetivos y desarrollo de la intervención}

El proyecto tuvo por objetivos: a) diseñar y ejecutar talleres de sensibilización y formación para equipos sanitarios sobre buenas prácticas para servicios de salud de calidad, inclusivos y accesibles para las personas en situación de discapacidad, con enfoque de derechos humanos y género; b) difundir el marco normativo y conceptual vigente acorde a los consensos internacionales, regionales y nacionales sobre discapacidad, derechos humanos, género y salud sexual y reproductiva; c) promover la reflexión entre profesionales sanitarios y de gestión en salud sobre las temáticas antes mencionadas; d) conocer el estado de situación relativo al acceso a servicios y a la calidad de atención que informan los usuarios en situación de discapacidad; e) aproximarse a las experiencias de los equipos de salud vinculadas al trabajo directo con usuarios en situación de discapacidad; f) relevar las principales necesidades y demandas sobre salud sexual y reproductiva que tienen los usuarios, así como también, las ideas previas con las que cuentan los equipos de salud; g) brindar herramientas teórico/conceptuales que se traduzcan en buenas prácticas profesionales en el campo de la salud.

La intervención consistió en el diseño, implementación y evaluación de actividades de formación y sensibilización sobre el "acceso universal a servicios de salud inclusivos y de calidad" dirigidos a: a) personas usuarias en situación de discapacidad; b) profesionales de la salud, con énfasis en los equipos de salud sexual y reproductiva; y c) personal de gestión de los servicios. La intervención tuvo lugar en el segundo semestre del 2019. En una primera instancia, la propuesta fue diseñada para su implementación en el subsector público de la salud y posteriormente se amplió al subsector privado.

Se destaca que los talleres eran instancias únicas, presenciales, de cuatro horas de duración y con un proceso de inscripción previo en el que se solicitaba una serie de datos que posteriormente permitiera construir el perfil de los participantes. Asimismo, en el marco de los talleres, es posible identificar dos momentos de trabajo diferenciados, uno de exposición teórica-conceptual y otro de trabajo práctico-vivencial; este último comprende, a su vez, un trabajo subgrupal y el intercambio posterior de cierre en el grupo general.

Las tareas de planificación, ejecución y análisis de la intervención estuvieron a cargo de docentes de la Facultad de Psicología de la Universidad de la República (UdelaR) a través del Instituto de Psicología de la Salud (Programa género, sexualidad y salud reproductiva) y el Instituto de Fundamentos y Métodos en Psicología (Programa discapacidad y calidad de vida), con la colaboración de docentes del Instituto Superior de Educación Física (ISEF) y de la Facultad de Medicina.

Las actividades contemplaron los ajustes necesarios para garantizar la participación a través de material con diseño universal, espacios físicos accesibles e intérprete de lengua de señas uruguaya.

La intervención se organizó en una primera fase (fase 1) en la que se convocó a personas y organizaciones sociales vinculadas a la temática con el fin de conocer sus demandas de atención en salud. Para ello se realizaron dos talleres, uno en la ciudad de Montevideo y otro en la ciudad de Paysandú. Se contó con la participación personas en situación de discapacidad, familiares, cuidadores, asistentes personales, intérpretes de lengua de señas, referentes institucionales de organizaciones sociales e instituciones estatales.

En una siguiente fase (fase 2) se realizaron cuatro reuniones de trabajo con metodología de taller con equipos de salud de cuatro ciudades de Uruguay logrando, a través de esta selección, una representatividad 
territorial en base a la segmentación geográfica utilizada por la Administración de los Servicios de Salud del Estado (ASSE) para sus regionales.

Estos talleres se organizaron en dos bloques, uno de formación y otro de sensibilización. El primero incluyó abordar los conceptos teóricos de derechos humanos, discapacidad (definición, modelos, terminología consensuada, diseño universal), género (definición, estereotipos de género, modelos hegemónicos de masculinidad y feminidad, violencia basada en género), salud sexual y reproductiva (historia, normativa nacional e internacional, derechos sexuales y reproductivos), sexualidad y diversidad sexual (definiciones, modelo hegemónico de sexualidad, fines de la sexualidad, enfoques de sexualidad, orientación sexual, identidad de género y expresión de género), interseccionalidad y herramientas para la atención a la salud sexual y reproductiva de las personas en situación de discapacidad en los servicios de salud.

El segundo se organizó en subgrupos en los que se trabajó en base a viñetas clínicas (tabla 1) que presentaban situaciones reales, anonimizadas, de personas en diversas situaciones de discapacidad sobre la atención en los servicios de salud y la vulneración de sus derechos como usuarios.

Las preguntas que consignaron el trabajo subgrupal fueron: ¿Es una situación de discapacidad? ¿Por qué? ¿Existe un problema en dicha situación? ¿Cuál es el problema principal que se detecta en la situación? ¿Qué otra acción podría haberse realizado desde el equipo de salud? y pensando en los lugares en los que trabajan: ¿Qué acciones específicas se pueden realizar para garantizar la atención de la persona en situación de discapacidad considerando sus derechos?

Tabla 1. Ejemplos de viñetas clínicas utilizadas en la segunda fase de la intervención

\begin{tabular}{|c|c|}
\hline Viñeta 1 & $\begin{array}{l}\text { Joaquín está esperando con su papá que lo atienda el médico que lo conoce desde que nació. Si bien tienen } \\
\text { una hora asignada, la consulta está atrasada media hora. } \\
\text { Joaquín no tolera esperar; y cuando se pone nervioso sube el volumen de su voz y camina por el lugar. Las } \\
\text { personas que también están esperando comienzan a ponerse impacientes. La administrativa de la sala se } \\
\text { acerca al papá de Joaquín y le ofrece otra hora para consultar. El papá acepta y se retiran de la sala con } \\
\text { Joaquín. }\end{array}$ \\
\hline Viñeta 2 & $\begin{array}{l}\text { Laura tiene } 24 \text { años. Su hermana, de } 16 \text { años, fue al ginecólogo por primera vez y lo que le contó despertó } \\
\text { interés en Laura; ella también quiere ir. Si bien cada tres meses va al médico con su mamá, hay preguntas que } \\
\text { le da vergüenza hacer delante de ella y de su médico, que es varón. Laura necesita alguien que sepa lengua } \\
\text { de señas uruguaya la acompañe ya que no lee labios. Cuando Laura le cuenta a su hermana esta situación, su } \\
\text { hermana se ofrece para hacerle a su ginecólogo las preguntas que Laura no se anima a hacer. }\end{array}$ \\
\hline Viñeta 3 & $\begin{array}{l}\text { Federico, de } 24 \text { años, reside junto con sus padres y hace dos años les manifestó que se siente mujer y que le } \\
\text { gustaría llamarse Rosalinda. Tras idas y vueltas, sus padres deciden acompañarle a una consulta médica. En } \\
\text { medio de la consulta, el profesional le dice a sus padres que es imposible que el usuario sepa lo que quiere a } \\
\text { causa de su discapacidad intelectual, y les comunica que no apoyará el proceso de reafirmación de género. } \\
\text { Federico se retira del servicio de salud sumamente angustiado y sus padres realmente desconcertados. }\end{array}$ \\
\hline Viñeta 4 & $\begin{array}{l}\text { Antonio y Carmen son pareja desde hace } 5 \text { años, están viviendo juntos desde hace } 2 \text { meses y van a tener un } \\
\text { hijo; como se mudaron de casa tuvieron que cambiar de centro de salud para realizar sus consultas y retirada } \\
\text { de medicación. Al llegar a la puerta de la policlínica de su zona, cerca de su casa, si bien hay una rampa, sus } \\
\text { sillas no pasan por la puerta. El médico los atiende amablemente en un consultorio con una entrada alternativa } \\
\text { por esta vez pero les sugiere que la próxima consulta la realicen en un hospital céntrico donde es seguro que } \\
\text { pueden ingresar a las instalaciones. }\end{array}$ \\
\hline
\end{tabular}

Fuente: elaboración propia. 
La información obtenida durante las actividades desarrolladas se documentó en diarios de campo realizados y actualizados por los talleristas y los coordinadores de la propuesta. En las bitácoras se registraron las observaciones vinculadas al lenguaje verbal, no verbal y paraverbal de los participantes para su posterior análisis.

La elección de no restringir la participación a estos espacios únicamente a quienes trabajan en atención directa sino de hacerla lo más abarcativa posible se relaciona con la necesidad de abordar la temática con todos los actores que estén relacionados: desde lugares de decisión y con posibilidad de cambios de protocolos hasta quienes tienen contacto directo con las usuarias y usuarios atendidos. Desde el equipo que coordinó e implementó estos talleres se entiende que la formación en salud sexual y reproductiva, en interrelación con la situación de discapacidad, debe ser transversal a todos estos actores.

En cuanto a los participantes, se registró un total de 236 personas, correspondiendo un 96,19\% a mujeres. Se puede observar que, si bien la convocatoria fue abierta, la menor participación se relaciona con áreas de gestión sin registrarse de dirección o gerencias. Por el contrario, más de un 70 \% corresponde a trabajadores del área de la salud de diversas disciplinas entre las que se contó con las siguientes (nombradas según orden de participación): enfermería, medicina, partería, psicología, trabajo social, fonoaudiología, fisioterapia y terapia ocupacional. La distribución por subsector también fue equitativa, habiendo participantes de un solo subsector (en su mayoría público, con un $47 \%$, y $32 \%$ en el sector privado), como también el $21 \%$ de trabajadores de ambos subsectores.

\section{Resultados y discusión}

En todos los talleres participaron personas o que contaban con conocimientos previos sobre derechos sexuales y reproductivos pero no sobre la temática de la discapacidad, la situación era inversa o en algunos casos no se contaba con conocimientos de ninguna de las dos temáticas. Esto implicó que el equipo fundamentalmente se centrara en una nivelación de saberes para posteriormente poder introducir la intersección de las dos temáticas centrales del proyecto. A partir de lo surgido en los talleres, y en base al principio de saturación de la información, se identificaron los siguientes emergentes para el análisis de la experiencia.

\section{- Relación con las personas usuarias en situación de discapacidad}

Los profesionales manifestaron en ocasiones no saber cómo dirigirse a ellas y a sus familiares, así como también, desconocían los procedimientos correctos para intervenir sobre sus cuerpos sin violentarlas. En esta línea, se consensuó que lo mejor era consultarles directamente. Esta duda es frecuente en la población general, y específicamente en los técnicos que trabajan en sectores de la salud sexual y reproductiva. Estas actitudes inciden directamente en los momentos de atención, derivación, orientación y comunicación específica de temas o asuntos que son de importancia para las personas usuarias y sus familiares. Se percibe, en los discursos de los y las participantes que existe una representación infantilizada de las personas en situación de discapacidad, especialmente de aquellas que requieren de acompañante. 
Al comienzo de las instancias las personas participantes de los talleres tienden a responsabilizar a los usuarios y usuarias con discapacidad por la existencia de las dificultades al momento del acceso a la salud, pero, tras las instancias de intercambio, se lo adjudican a situaciones de discriminación y exclusión. Algunos de los profesionales de la salud sugirieron que conocer con anterioridad el "diagnóstico de discapacidad" puede permitir que las personas usuarias reciban la atención requerida ya que la institución pondría a disposición recursos específicos para compensar esas dificultades. En ocasiones los propios profesionales expresan que las instituciones se caracterizan por tener barreras edilicias y actitudinales que se vuelven discapacitantes para los sujetos porque limitan su autonomía y reproducen desigualdades con relación al resto de usuarios de los servicios.

\section{- Ejercicio de derechos sexuales y reproductivos}

Se constata una creencia generalizada que niega la condición de sujetos sexuados de las personas en situación de discapacidad. En los casos de diagnósticos congénitos esta creencia se mantiene activa durante todo el ciclo de vida, mientras que, en los casos de discapacidades adquiridas pareciera que los usuarios y usuarias devienen en sujetos asexuales. Esto produce que desde los prestadores no se ofrezcan ni faciliten servicios de salud sexual y reproductiva. Otras vulneraciones se producen debido al desconocimiento de la normativa y reglamentación vigente sin respetar el principio de la autonomía progresiva o los espacios de confidencialidad en el marco de una consulta cuidada, ya que, los adolescentes generalmente no ingresan solos a consulta o los adultos ingresan con sus acompañantes.

Aquellos profesionales que sí habilitan cuestiones vinculadas a la sexualidad acaban reproduciendo el modelo heteronormativo; por ende, es un impensable que las personas usuarias sean gays, lesbianas o bisexuales, o que directamente el motivo de consulta pueda estar vinculado al desarrollo de una identidad trans y a la necesidad de iniciar un tratamiento de hormonización que facilite la reafirmación de género; estas situaciones se vuelven aún más significativas cuando se trata de una discapacidad intelectual o psíquica.

Desde las narrativas de los profesionales, también se constatan una serie de omisiones en el primer nivel de atención relativas a la promoción de salud sexual y reproductiva como, por ejemplo, no brindar información sobre prevención de abusos sexuales, a pesar de que se tiene conocimiento de que es un problema prevalente en situaciones de discapacidad intelectual, psíquica y auditiva, tanto en varones como en mujeres.

Asimismo, se observan dificultades para visibilizar los deseos y posibilidades de maternidad y paternidad de estas personas; siendo otro ejemplo de vulneración de derechos reproductivos, en lo relativo a la planificación familiar. Se plantearon situaciones de violencia obstétrica en instancias de partos naturales y cesáreas (especialmente en casos de discapacidad auditiva), así como también la existencia de que profesionales especialistas en ginecología no indican el test de Papanicolaou, partiendo del supuesto de que las mujeres en situación de discapacidad no son sexualmente activas.

Una de las preguntas frecuentes por parte de los familiares directos de usuarios varones, es sobre las prácticas masturbatorias. Generalmente las familias le inhiben la vida autoerótica a los usuarios porque consideran que es contraproducente para su salud. En este sentido, personas con discapacidades físicas moderadas y severas habitualmente denuncian la falta de espacios íntimos donde puedan explorar su vida autoerótica con autonomía y libertad. Por otro lado, los usuarios insistieron en que las redes sociales online les han otorgado nuevas posibilidades para ejercer activamente su sexualidad. 


\section{- Accesibilidad institucional}

Al plantear a las personas participantes que problematizaran este aspecto concreto de las barreras para la inclusión, se logran identificar problemas específicos: falta de disponibilidad de un intérprete de lengua de señas uruguaya, falta de accesibilidad arquitectónica para el ingreso en las instituciones o la transitabilidad interna para hacer uso de los servicios necesarios, falta de mobiliario específico actualizado como, por ejemplo, las camillas, no cuentan con las características necesarias para ser consideradas accesibles.

\section{Consideraciones finales}

Se describen en este apartado una serie de observaciones que pretenden hacer visibles las necesidades en lo que se refiere a las herramientas y saberes acumulados de los equipos de salud, así como sus potencialidades, a fin de mejorar la atención de usuarias y usuarios con o sin discapacidad, en clave de derechos humanos.

En relación a los datos obtenidos de quienes participaron, se destaca que trabajan desde lo interprofesional, en el primer nivel de atención o en áreas disciplinares menos especializadas. En relación a la distribución de estos según la formación, los cargos (técnicos y administrativos), así como la edad y el género, tienen relación con la proporción de los mismos en las diversas áreas de trabajo público y privado.

En este sentido, se entiende fundamental visualizar la necesidad de que, como sociedad, Uruguay pueda problematizar acerca de la forma en que se desarrolla la atención de la salud, visualizando una transformación tendente hacia la atención integral, la producción dialógica de salud y la clínica ampliada con un enfoque interprofesional.

Una de las dificultades encontradas es la baja participación de trabajadores y trabajadoras que ejercen cargos de dirección; se entiende que está asociado a esta concepción de que quienes deben implementar las acciones inclusivas propuestas son quienes trabajan directamente con las personas usuarias, no reconociendo que es fundamental para el éxito de éstas el diseño de protocolos, servicios con ajustes y el respaldo institucional para el desarrollo de las mismas.

La discusión entre los participantes se dio en relación a los siguientes temas: identificación de limitación/ discapacidad, barreras comunicacionales, barreras actitudinales, barreras arquitectónicas, barreras institucionales, accesibilidad a la salud sexual y reproductiva, y propuestas de acciones superadoras y soluciones alternativas.

En este sentido, y como primera observación, se visualiza una importante diversidad de situaciones en cuanto a los saberes de las personas participantes, impactando directamente sobre el diseño metodológico propuesto de las instancias presenciales. Se sugiere dar continuidad a este proceso de trabajo con capacitaciones centradas estrictamente en esta intersección.

La anterior situación planteada dejó sin efecto el objetivo de "relevamiento y propuesta de prácticas, avances y mejoras ya implementadas o posibles a desarrollar", si bien en muchos casos se conoce la existencia 
de leyes, se desconoce su vigencia, su contenido y su reglamentación. La discusión y el intento de síntesis de estos aspectos consumieron un gran tiempo de trabajo. Desde los participantes se plantea que hay necesidades (arquitectónicas, materiales, presupuestales y de recursos humanos) que se deben resolver de manera previa a la implementación de intervenciones inclusivas.

En muchos casos se visualizan perspectivas predominantemente biomédicas en lo referido a la intervención y la acción sanitaria. Si bien desde el discurso la mayoría de las personas participantes se autodefinen desde una perspectiva de derechos, se visualiza una tendencia a concebir la discapacidad desde una perspectiva biomédica ligada a la enfermedad.

A partir de los intercambios con usuarios y con profesionales integrantes de los equipos de salud es posible afirmar que el acceso a la atención de la salud sexual y reproductiva por parte de las personas en situación de discapacidad presenta dificultades y por ende el ejercicio de ciudadanía de este colectivo es restringido. Pareciera que la sexualidad de las personas en situación de discapacidad aún es un tabú y que la categoría de sujetos sexuados es invisibilizada, negada y silenciada por parte de los equipos de salud, todo lo cual impacta en la posibilidad real de ejercicio del conjunto de derechos sexuales y reproductivos.

Creemos pertinente que se pueda realizar un seguimiento de los profesionales capacitados con el fin de recabar sus percepciones a la hora de implementar o incorporar los conocimientos adquiridos. Así mismo sería indicado recabar acciones concretas de "buenas prácticas" que puedan ser tomadas como ejemplo para incorporar en otras capacitaciones y formaciones. En este sentido se recomienda recabar aspectos como: posibles tensiones entre las definiciones y/o sugerencias teóricas y las posibilidades de llevarlas a la práctica, vacío de saberes, lo que se desconoce de lo normativo, interseccionalidad, especificidad de la discapacidad y posibles ajustes.

Para finalizar, si bien no se hizo una evaluación escrita de los talleres de sensibilización y capacitación, se recogieron las opiniones de las personas participantes al finalizar la actividad. Las mismas expresaron un profundo agradecimiento por este tipo de instancias, tanto en lo que refiere a la capacitación, como a la posibilidad de intercambiar experiencias con otros pares de distintas regiones. Resaltaron lo rico de trabajar con viñetas que representan situaciones de la vida cotidiana y, de este modo, darse el tiempo de reflexionar sobre cómo actuar y no solo actuar, como generalmente les ocurre en el día a día.

Sin embargo, también resaltaron - en cada uno los talleres - la necesidad de un apoyo y compromiso en la temática desde las direcciones políticas nacionales y departamentales. Un ejemplo que apareció en todas las instancias fue la incapacidad por parte del equipo técnico de conseguir intérpretes de lengua de señas, ya que la(s) institución(es) no cuentan con los mismos y muchas veces no se sabe cómo gestionar su contratación de forma puntual y concreta. 
Referencias bibliográficas

Instituto Nacional de Estadística de Uruguay (2011). Censo de población y vivienda. https://www.ine.gub.uy/web/ guest/censos-2011.

López, A. (2013). Salud Sexual y Reproductiva en la agenda de investigación y formación en Psicología en Uruguay. Conferencia inaugural actividades académicas 2013, Facultad de Psicología, Universidad de la República, Montevideo, Uruguay. http://psico.edu.uy/sites/default/files/files_ftp/libros/conferencia-2013.pdf.

López, P. et al. (2015). Salud y diversidad sexual: guía para profesionales de la salud. UNFPA.

Mattioli, N. (Coord.) (2012). Guías en Salud Sexual y Reproductiva: Abordaje de la Salud Sexual y Salud Reproductiva en personas con discapacidad. Ministerio de Salud Pública. https://www.paho.org/uru/index. php?option=com_docman\&view=download\&category_slug=promocion-de-salud\&alias=381-libro-discapacidad-ssr-2012\&ltemid=307.

Ministerio de Salud Pública (2010). Guías para implementar servicios de atención a la salud sexual y salud reproductiva en las instituciones prestadoras de salud. Ministerio de Salud Pública. https://uruguay.unfpa.org/sites/ default/files/pub-pdf/12_file2.pdf.

Ministerio de Salud Pública (8 de enero de 2019). Objetivos Sanitarios Nacionales 2020. Ministerio de Salud Pública. Recuperado el 9 de julio de 2020 de https://www.gub.uy/ministerio-salud-publica/comunicacion/publicaciones/publicaciones-objetivos-sanitarios-nacionales-2020.

Nagai, A. y Pereira, J. (2017). Sexualidade das pessoas com deficiência: uma revisão sistemática. Arquivos Brasileiros de Psicologia, 70(3), pp. 289-304. http://pepsic.bvsalud.org/pdf/arbp/v70n3/20.pdf.

Organización de las Naciones Unidas (2006). Convención sobre los derechos de las personas con discapacidad. ONU. https://www.un.org/esa/socdev/enable/documents/tccconvs.pdf.

Organización Mundial de la Salud (2011). Informe Mundial sobre la discapacidad. OMS. https://www.who.int/ disabilities/world_report/2011/es/.

Organización Panamericana de la Salud y Organización Mundial de la Salud (2013). Por la salud de las personas trans. Elementos para el desarrollo de la atención integral de personas trans y sus comunidades en Latinoamérica y El Caribe. OPS- OMS. https://pdf.usaid.gov/pdf_docs/PA00JQ81.pdf.

Palacios, A. (24-25 de mayo de 2016). La configuración de los sistemas de apoyo en el contexto de la accesibilidad universal y los ajustes razonables. Congreso internacional Madrid sin barreras: accesibilidad, ajustes y apoyos. Universidad Carlos III de Madrid. https://www.ohchr.org/Documents/HRBodies/CRPD/EqualityNonDiscrimination/presentations/AgustinaPalaciosCONICET.docx.

Registro Único de Cobertura de Asistencia Formal (RUCAF) (9 de agosto de 2018). Informe cobertura poblacional del SNIS según prestador 2017-2018. Ministerio de Salud Pública. Recuperado el 9 de julio de 2020 de https:// www.gub.uy/ministerio-salud-publica/comunicacion/publicaciones/informe-cobertura-poblacional-del-snissegun-prestador-2017-2018.

Rudolf, S. et al. (2009). Accesibilidad y participación ciudadana en el sistema de salud. Una mirada desde la psicología. Fin de Siglo. 
Uruguay. Ley N 18.211/2007, de 5 de diciembre, Creación del Sistema Nacional Integrado de Salud. Diario Oficial, 13 de diciembre de 2007, núm. 27384.

Uruguay. Ley $N^{\circ}$ 18.335/2008, de 15 de agosto, Derechos y obligaciones de pacientes y usuarios de los servicios de salud. Diario Oficial, 26 de agosto de 2008, núm. 27554.

Uruguay. Ley $\mathrm{N}^{\circ} 18.426 / 2008$, Defensa del derecho a la salud sexual y reproductiva. Diario Oficial, 10 de diciembre de 2008, núm. 27630 .

Uruguay. Ley $N^{\circ}$ 18.651/2010, Protección integral de personas con discapacidad. Diario Oficial, 9 de marzo de 2010, núm. 27932.

Uruguay. Ley N¹8.987/2012, Interrupción voluntaria del embarazo. Diario Oficial, 30 de octubre de 2012, núm. 28585.

Vignolo, J. et al. (2011). Niveles de atención, de prevención y atención primaria de la salud. Archivos de Medicina Interna, 33(1), pp. 7-11. 